\title{
Determining Malignancy of Brain Tumors by Analysis of Vessel Shape
}

\author{
Elizabeth Bullitt ${ }^{1}$, Inkyung Jung ${ }^{2}$, Keith Muller ${ }^{2}$, Guido Gerig ${ }^{3}$, Stephen Aylward ${ }^{4}$, \\ Sarang Joshi ${ }^{5}$, Keith Smith $^{4}$, Weili Lin ${ }^{4}$, and Matthew Ewend ${ }^{1}$ \\ ${ }^{1}$ Departments of Surgery, \\ ${ }^{2}$ Biostatiscs, \\ ${ }^{3}$ Computer Science, \\ ${ }^{4}$ Radiology, and \\ ${ }^{5}$ Radiation Oncology, University of North Carolina, Chapel Hill, NC, 27599, USA \\ (bullitt, jksmith, weili_lin, ewend\} @med.unc.edu, \\ $\{$ keith_muller, aylward\} @unc.edu, \\ \{gerig, joshi\}@es.unc.edu
}

\begin{abstract}
Vessels supplying malignant tumors are abnormally shaped. This paper describes a blinded study that assessed tumor malignancy by analyzing vessel shape within MR images of 21 brain tumors prior to surgical resection. The program's assessment of malignancy was then compared to the final histological diagnosis. All tumors were classified correctly as benign or malignant. Of importance, malignancy-associated vessel abnormalities extend outside apparent tumor margins, thus allowing classification of even small or hemorrhagic tumors.
\end{abstract}

\section{Introduction}

Despite the development of new imaging techniques, noninvasive determination of tumor malignancy remains a difficult task [1], [2], [3], [4]. An intriguing observation made by those working from histological section is that blood vessels associated with malignant tumors exhibit characteristic shape abnormalities. As stated by Baish, such vessels display a " .... a profound sort of tortuosity, with many smaller bends upon each larger bend" [5]. This abnormal tortuosity may be related to increases in nitrous oxide induced by $\mathrm{VegF}$ [6], and is found in many malignant tumors including those of the breast [7], brain [8], colon [9], and lung [10]. Moreover, changes in vessel tortuosity precede sprout formation, thus providing a potential marker for incipient malignancy [6]. Of equal importance, successful tumor treatment normalizes vessel shape [11]. All of the above observations were made from histological section. If one could quantify such vessel shape changes from in vivo medical images, the approach could offer a new and potentially powerful means of estimating tumor malignancy and of monitoring treatment response noninvasively. Indeed, indirect measurements of vessel tortuosity within in vivo images have already been described by Jackson et al., who note that variation in the recirculation characteristics of a contrast agent bolus is related to 
the tumor grade of gliomas, and may represent vascular tortuosity and hypoperfusion in areas of angiogenic neovascularization [12].

The current study originates from an accidental observation. Our group has focused upon the segmentation of vessels and tumors from magnetic resonance (MR) images. About two years ago, our vascular imaging protocols were upgraded to provide submillimeter voxel spacing. Shortly thereafter, we noticed that vessels segmented from the vicinity of malignant tumors appeared abnormally "wiggly". Since then, we have developed metrics to quantify the tortuosity of segmented vessels [13], and have performed a pilot study to define shape measures likely to be useful in characterizing vessels of malignancy [14].

This paper describes a blinded study that used measures of vessel shape to diagnose malignancy from images of twenty-one brain tumors in patients scheduled for tumor removal. The goal was to noninvasively discriminate malignant from benign tumors including cases difficult to classify by other imaging methods. Many "difficult" cases were included, such as hemorrhagic lesions, irradiated tumors, pinpoint abnormalities, and hypervascular benign lesions. For each tumor, the program's classification of benign or malignant was compared to the final pathological diagnosis. All twenty-one tumors were classified correctly. A surprising and significant finding was that the vessel shape changes associated with malignancy seem to extend well outside of apparent tumor margins. Although this report analyzes only MR images of the brain, the method is extensible to vascular images of any anatomical location.

\section{Background}

Detecting abnormal tortuosity within the intracerebral circulation is difficult because healthy intracerebral vessels are ordinarily tortuous. Most of the previous work on defining vessel tortuosity has been performed in 2D, focusing upon retinopathy of prematurity. The most common metric is the "Distance Metric" which provides the total path length of a vessel divided by distance between endpoints. Brey [15] extended this metric to 3D for analysis of vessels in histological section. A problem with the Distance Metric, however, is that it assigns a higher value to a healthy, long, "S" shaped vessel than to a short, abnormal vessel with tight coils. Baish [5] and Sabo [16] are attempting to stage tumor malignancy on the basis of vessel tortuosity within histological sections by using microvessal fractal dimension.

The current study takes a different approach, and assumes that the clinical assessment of "abnormal tortuousity" can refer to more than one type of shape abnormality. Webster's dictionary defines "tortuosity" as "full of twists, turns; crooked" [17]. However, what precisely is meant by "tortuosity" in a medical sense is unclear.

One can view "abnormal tortuosity" as comprising three different patterns [13]. Tortuosity type I occurs when a straight vessel elongates to form a " $\mathrm{C}$ " or " $\mathrm{S}$ ", and occurs with retinopathy of prematurity, anemia, and hypertension. Tortuosity type II is 
characterized by a "bag of worms" configuration, and occurs within many hypervascular tumors and arteriovenous malformations. Tortuosity type III is characterized by high-frequency, low-amplitude coils or sine waves, and appears within malignant tumors.

We have previously described two 3D tortuosity metrics that act upon sets of segmented vessels [13]. The Inflection Count Metric (ICM) counts loci of minimum curvature along a 3D space curve, multiplies this number (plus 1) times the total path length of the curve, and divides by the distance between endpoints. The ICM detects abnormal tortuosity types I and II, but fails with type III. The second method, the Sum Of Angles Metric (SOAM), measures the curvature along a space curve and normalizes by total path length. The SOAM detects abnormal tortuosity type III, but fails with types I and II. Malignant tumor vessels, which exhibit "many smaller bends upon each larger bend" [5], tend to display a mix of tortuosity types II and III, and thus usually show increases in tortuosity as measured by both by SOAM and ICM. Figure 1 illustrates the typical tortuosity pattern of vessels within a malignant tumor.
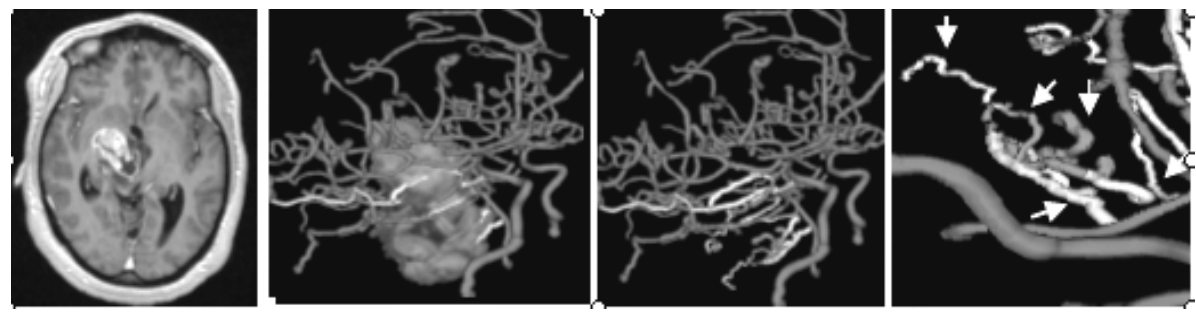

Fig. 1. Typical vessel shape changes with malignancy. Left: T1, gadolinium enhanced section of a glioblastoma. Central images: Vessels color-coded with relationship to the tumor surface and with the tumor shown at full and at no opacity. Right: Magnification of tumor vessels. Arrows point to abnormal, high-frequency wiggles (tortuosity type III, detectable by SOAM). Many of the vessels also have somewhat of a "bag of worms" appearance (tortuosity type II, detectable by ICM).

\section{Methods}

\subsection{Patient Selection and Image Acquisition}

Images of sixteen healthy subjects, ranging in age from twenty-two to fifty-four, were used to establish the healthy database. Tumor cases included twenty-one brain lesions in nineteen patients, each scheduled for total gross resection of their tumor or tumors.

Images were obtained upon a head-only 3T MR unit (Allegra, Siemens Medical Systems Inc., Germany) or upon a 1.5T MR unit (Sonata, Siemens Medical Systems Inc., Germany). A quadrature head coil was employed. T1, T2, and MRA sequences were performed on all subjects, with tumor patients additionally receiving a gadolinium enhanced $\mathrm{T} 1$ sequence. 
Vascular images employed a 3D time-of-flight MRA sequence. Velocity compensation along both frequency and phase encoding directions was used to minimize signal dephasing induced by the flowing spins. In addition, a magnetization transfer pulse was employed to suppress signal from brain parenchyma while maintaining signal from flowing spins. Images were obtained at 512 x 512 x $\sim 120$. Voxel spacing was $0.5 \times 0.5 \times 0.8 \mathrm{~mm}^{3}$ in the majority of cases (our current standard) but some early patients were scanned at $0.8 \times 0.8 \times 1.0 \mathrm{~mm}^{3}$ or at $0.4 \times 0.4 \times 1.25 \mathrm{~mm}^{3}$.

\subsection{Image Processing}

Vessel segmentation was done by Aylward's method [18]. Vessel extraction involves 3 steps: definition of a seed point, automatic extraction of an image intensity ridge representing the vessel's central skeleton, and automatic determination of vessel radius at each skeleton point. The output of the program provides sets of directed, 4dimensional points indicating the $(\mathrm{x}, \mathrm{y}, \mathrm{z})$ spatial position of each sequential vessel skeleton point and an associated radius at each point. Extracted vessels were then postprocessed to produce connected vessel trees and to exclude noise [19].

Tumor segmentation was performed using either a fully automated method [20] or a partially manual program that segments tumors via polygon drawing and filling on orthogonal cuts through an image volume (http://www.cs.unc.edu/ gerig/).

All images were registered via a mutual-information based, affine transformation [21], [22] into the coordinate system of the McConnell atlas [23] so that, via a combination of forward and backward mapping, the same region of interest could be defined within each image. For each tumor patient, vessels were automatically clipped to the tumor margins and an automated analysis of vessel shape was performed only upon those vessels lying within the tumor boundaries. This same region of interest was then mapped into the coordinate space of each healthy patient, and a similar, regional analysis was performed upon each healthy subject's vasculature.

Three vessel attributes of interest were defined during an earlier pilot study [14], and consist of one vessel density measure and the two tortuosity metrics discussed earlier. The terminal branch count (TBC) counts the number of vessels entirely contained within a region of interest. As described earlier, the SOAM is a tortuosity measure effective in detecting high frequency sine waves and tight coils, and the ICM is a tortuosity measure effective in detecting "bag of worms" tortuosity [13].

Tiny or hemorrhagic lesions may contain no vessels visualizable by MRA. Our approach requires some minimum number of vessels for analysis. We arbitrarily decided upon a minimum vessel number of 4 . If an insufficient number of vessels were present within the tumor boundary as initially defined, we therefore expanded this boundary to include the edematous region around the tumor. For lesions without surrounding edema, the "tumor boundary" was dilated until it included 4 vessels. It takes 60-90 minutes to process each new case. 


\subsection{Blinded Study and Subsequent Discriminant Analysis}

Nineteen patients with a total of twenty-one tumors were recruited, imaged by MR, and operated upon by a single surgeon. Image analysis was performed by a different individual who was blinded to the patient's history and histology until after the analysis was completed and reported to the surgeon. Each tumor was declared benign or malignant with results evaluated by comparison to the final histological diagnosis.

We entered into this study with the belief that high-frequency wiggles detectable by SOAM were likely to represent the single most important measure of vessel shape. Any tumor whose SOAM value was elevated by two or more standard deviations from the healthy mean was thus automatically defined as malignant. Any tumor whose SOAM value lay between one and two standard deviations above the healthy mean was viewed as suspicious, and the tumor was then declared malignant if the secondary ICM value lay more than two standard deviations above the healthy mean.

After study completion, a formal statistical analysis was conducted of the twentysix tumors (five in the pilot study and twenty-one in the blinded study) to investigate whether a purely quantitative rule could be formulated that would automate diagnosis. Each score was standardized relative to the mean and standard deviation for values in the corresponding region of interest of the healthy subjects. The exploratory analysis led to concerns about assuming a Gaussian distribution or common covariance. A quadratic discriminant analysis and a nonparametric discriminant analysis were therefore performed.

\section{Results}

Of the twenty-one tumors, twelve were malignant and nine were benign. All cases were classified correctly during the blinded study. Potentially difficult cases such as a hypervascular benign tumor, a "pinpoint" metastatic lesion, a brain abscess felt preoperatively to be a malignant glioma, and a large intracerebral hemorrhage arising from an obliterated metastatic melanoma were properly labeled. Four benign and three malignant lesions had been treated by surgery and radiation therapy months or years earlier. History of prior irradiation therefore did not appear to disrupt vessel analysis. Figure 2 shows the vessels associated with two lesions, one benign and the second malignant, present in the same patient.
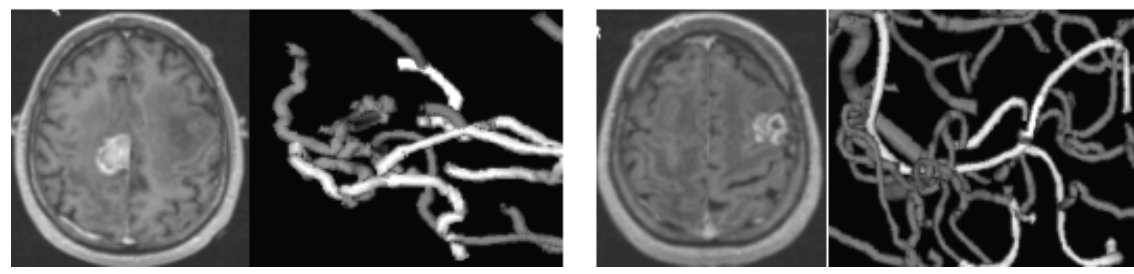

Fig. 2. Malignant tumor and associated vessels (left) and benign tumor with associated vessels (right) in the same patient. Note that the vessels associated with the malignant tumor have highfrequency wiggles, but that the vessels associated with the benign tumor are smooth. 
The study contained one example of a grade III glioma. Such tumors are malignant, but do not display the vascular proliferation associated with tumors of higher malignancy. Vessel analysis appropriately noted the terminal branch count to be within healthy range in this case, but also accurately declared the tumor to be malignant by the two vessel tortuosity measurements. This tumor is of interest, as its vascular pattern fits Folkman's observation that increased vessel tortuosity precedes sprout formation during malignant tumor growth ${ }^{6}$.

Although the majority of malignant tumors were hypervascular and the majority of benign tumors hypo- or normovascular, there was crossover in each group. Regardless of vessel density, malignancy was associated with vasculature bearing high-frequency tortuosity abnormalities detectable by SOAM. Tumors displaying these vascular changes included glioma grade IV, glioma grade III, lymphoma, metastatic melanoma, metastatic breast carcinoma, and pinealoblastoma.

Discriminant analysis, performed after completion of the prospective study, found the correlations between diagnosis ( 1 if malignant, 0 if benign) and the predictors to be $\{0.780 .51,0.30\}$ for $\{\mathrm{SOAM}, \mathrm{ICM}, \mathrm{TBC}\}$. Both quadratic and nonparametric discriminant rules classified all twenty-six patients correctly into the benign or malignant group using $\{\mathrm{SOAM}, \mathrm{ICM}, \mathrm{TBC}\}$. More specifically, the quadratic discriminant function is $\mathrm{Y}=.99 * \mathrm{SOAM}^{2}+3.58 * \mathrm{ICM}^{2}+.36 * \mathrm{TBC}^{2}-.95 * \mathrm{SOAM}^{2} \mathrm{ICM}+$ $.20 * \mathrm{SOAM} * \mathrm{TBC}-2.26 * \mathrm{ICM} * \mathrm{TBC}+.83 * \mathrm{SOAM}+7.05 * \mathrm{ICM}-2.09 * \mathrm{TBC}$, with the tumor malignant for $\mathrm{Y}>4.04$ and benign otherwise. The scores of malignant and benign tumors are separated, as shown by Figure 3.

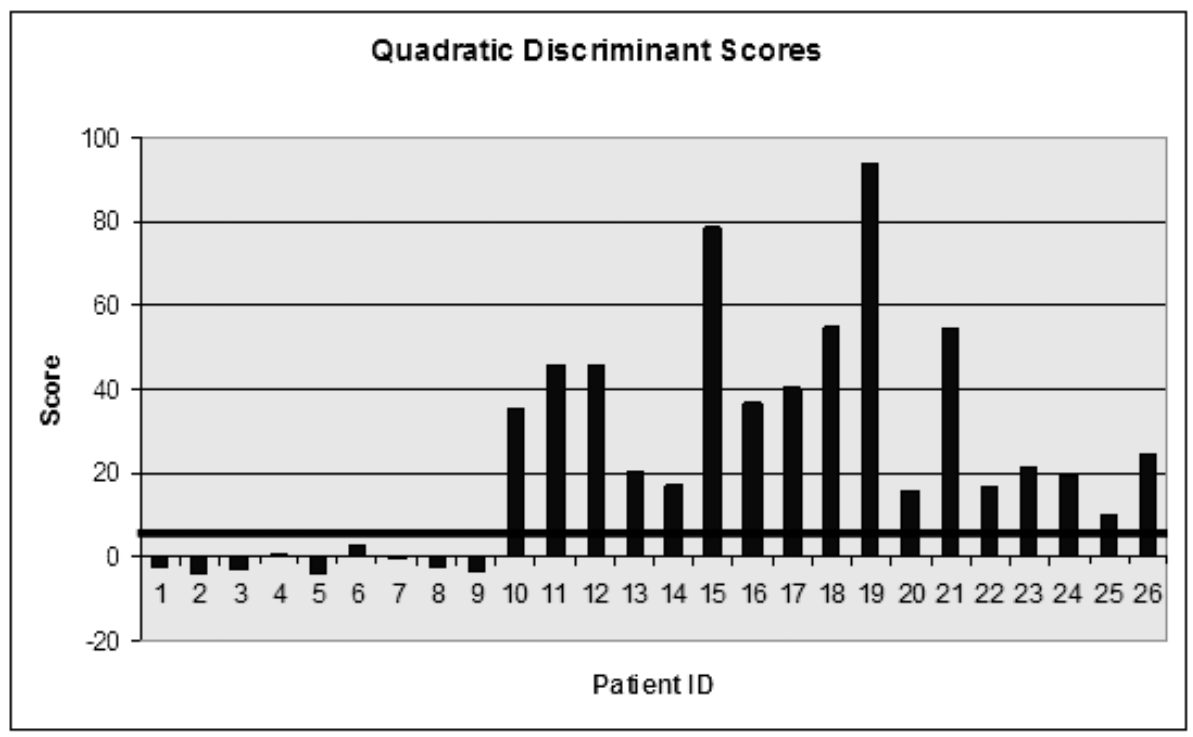

Fig. 3. Quadratic discriminant scores for 26 tumors. The first 9 tumors are benign and the remaining tumors are malignant. The thick black line is drawn through the cutoff point 4.04; all tumors with a lower score are benign and all tumors with a greater score are malignant. 


\section{Discussion}

This report describes a new, noninvasive method of determining tumor malignancy from high-resolution 3D images using a computerized analysis of vessel shape. Potential advantages over other described methods include the capacity to deal with hemorrhagic lesions, resilience to the necrosis produced by prior surgery or radiation treatment, handling of small lesions, and ability to distinguish between malignancy and hypervascular benign lesions. The number of cases analyzed here is small, however, and the formal equation produced by the final discriminant analysis will require testing in a much larger series.

At the outset of this study we hypothesized that different tumor types might possess distinctive vascular patterns. If this were the case, vessel analysis might help make a specific diagnosis (glioma $\mathrm{v}$ metastasis, for example). The series is too small and contains too many different malignant tumor types to permit significant comparisons between malignant subtypes. However, comparison of the four metastatic lesions to the eleven malignant gliomas indicates no obvious difference in vessel shape or distribution. If this observation holds for a larger series, it would support the concept of a single shape that characterizes "vessels of malignancy".

An important and surprising finding is that abnormal vessel tortuosity is found not only within the enhancing margins of malignant tumors but also in the surrounding tissue. How distantly these morphological changes extend is unknown, and is a topic for future research. An advantage of these relatively widespread changes is that even tiny lesions can be classified as malignant or benign via examination of vessels in the surrounding tissue.

Another potentially important finding relates to Folkman's observation that vessel tortuosity abnormalities precede sprout formation during malignant tumor growth [6]. Our series contains one case of a non-enhancing glioma grade III, which exhibited abnormal vessel tortuosity but no significant neovascularity. It therefore seems possible that vessel analysis could provide a means of early detection of incipient cancers. A longitudinal study would be needed to test this hypothesis.

A limitation of the approach is the requirement for high quality, high resolution vascular images. The vessels of interest are small and contain high-frequency, lowamplitude wiggles. Extension of the method to anatomical regions such as the lung is thus likely to require imaging techniques adapted to prevent blurring of vessels by respiratory motion.

Acknowledgments. This work was supported by R01 EB000219 NIBIB and HL69808 NIH-HLB. Portions of the software are licensed to Medtronic Corp (Minn., Minn) and to R2 Technologies (Los Altos, CA). We are grateful to Daniel Rueckert for his registration software. 


\section{References}

1. Benard F, Romsa J, Hustinx R: Imaging gliomas with positron emission tomography and single-photon emission computed tomography. Seminars Nuc. Med. 23: 148-162, 2003.

2. Burtscher LM, Holtas S: Proton magnetic resonance spectroscopy in brain tumors: clinical applications. Neuroradiology 43: 345-352, 2001.

3. Tosi MR, Fini G, Tinti A, Reggiani A, Tugnoli V: Molecular characterization of human healthy and neoplastic cerebral and renal tissues by in vitro ${ }^{1} \mathrm{H}$ NMR spectroscopy (Review). International Journal of Molecular Medicine 9: 299-310, 2002.

4. Law M, Yang S, Wang H, Babb JS, Johnson G, Cha S, Knopp EA, Zagzag D: Glioma Grading:Sensitivity, specificity, and predictive values of perfusion MR imaging and proton MR spectroscopic imaging compared with conventional MR imaging. AJNR 24:19891998, 2003.

5. Baish JS, Jain RK (2000) Fractals and cancer. Cancer Research 60:3683-3688, 2000.

6. Folkman J: Incipient Angiogenesis. Journal of the National Cancer Institute 92: 94-95, 2000 .

7. Lau DH, Xue L, Young LJ, Burke PA, Cheung AT: Paclitaxel (Taxol): an inhibitor of angiogenesis in a highly vascularized transgenic breast cancer. Cancer Biother. Radiopharm. 14:31-6, 1999.

8. Burger PC, Scheithauer BW, Vogel FS.: Surgical Pathology of the Nervous System and its Coverings, Third Edition, Churchill Livingstone, New York (1991).

9. Siemann D: Vascular Targeting Agents. Horizons in Cancer Therapeutics 3:4-15, 2002.

10. Helmlinger G, Sckell A, Dellian M, Forbes NS, Jain RK: Acid production in glycolysisimpaired tumors provides new insights into tumor metabolism. Clinical Cancer Research 8:1284-1291, 2002.

11. Jain RK : Normalizing tumor vasculature with anti-angiogenic therapy: a new paradigm for combination therapy Nature Medicine 7: 987-98, 2001.

12. Jackson A, Kassner A, Annesley-Williams D, Reid H, Zhu X, Li K: Abnormalities in the recirculation phase of contrast agent bolus passage in cerebral gliomas: Comparison with relative blood volume and tumor grade. AJNR 23:7-14, 2002.

13. Bullitt E, Gerig G, Pizer S, Aylward SR: Measuring tortuosity of the intracerebral vasculature from MRA images. IEEE-TMI 22:1163-1171, 2003.

14. Bullitt E, Gerig G, Aylward S, Joshi S, Smith K, Ewend M, Lin W: Vascular Attributes and Malignant Brain Tumors. MICCAI 2003; Lecture Notes in Computer Science 2878:671-679, 2003.

15. Brey EM, King TW, Johnston C, McIntire LV, Reece GP, Patrick CW: A technique for quantitative three-dimensional analysis of microvascular structure. Microvascular Research 63:279-294, 2002.

16. Sabo E, Boltenko A, Sova Y, Stein A, Kleinhaus S, Resnick MB: Microscopic Analysis and Significance of Vascular Architectural Complexity in Renal Cell Carcinoma. Clinical Cancer Research 7:533-537, 2001.

17. Neufeld, V. Webster's New World Dictionary. New York: Warner Books, 1990, p. 623.

18. Aylward $\mathrm{S}$, Bullitt $\mathrm{E}$ : Initialization, noise, singularities and scale in height ridge traversal for tubular object centerline extraction. IEEE-TMI 21:61-75, 2002.

19. Bullitt E, Aylward S, Smith K, Mukherji S, Jiroutek M, Muller K : Symbolic Description of Intracerebral Vessels Segmented from MRA and Evaluation by Comparison with XRay Angiograms. Medical Image Analysis 5:157-169, 2001.

20. Prastawa M, Bullitt E, Moon N, Van Leemput K, Gerig G: Automatic brain tumor segmentation by subject specific modification of atlas priors. Academic. Radiology 10:1341$1348,2003$. 
21. Schnabel JA, Rueckert D, Quist M, Blackall JM, Castellano Smith AD, Hartkens T, Penney GP, Hall WA, Liu H, Truwit CL, Gerritsen FA, Hill DLG, and Hawkes JD: A generic framework for non-rigid registration based on non-uniform multi-level free-form deformations. MICCAI 2001; Lecture Notes in Computer Science 2208: 573-581, 2001.

22. Rueckert D (2002) "Rview". Available: www.doc.ic.ac.uk/ dr/software.

23. ICBM Atlas, McConnell Brain Imaging Centre, Montréal Neurological Institute, McGill University, Montréal, Canada. 